\title{
Production in advance versus production to order
}

\author{
Attila Tasnádi ${ }^{1}$ \\ Department of Mathematics, Budapest University of Economic Sciences and \\ Public Administration, Fövám tér 8, H-1093 Budapest, Hungary \\ Appeared in the Journal of Economic Behavior and \\ Organization, 54(2004), 191-204, doi:10.1016/j.jebo.2003.04.005 \\ (C)Elsevier Science S.A.
}

\begin{abstract}
We use a theoretical framework to compare production-in-advance type and production-to-order type environments. Carrying out our analysis in the framework of a symmetric capacity-constrained Bertrand-Edgeworth duopoly game, we prove that the equilibrium profits are the same in case of production in advance and production to order. In addition, advance production results in higher prices than production to order if both games have an equilibrium in nondegenerated mixed strategies.
\end{abstract}

JEL classification: D43; L13

Keywords: Price-quantity games; Bertrand-Edgeworth competition.

\section{Introduction}

Shubik (1955) proposed that in a duopolistic context price-quantity games have to be considered besides price-setting games. He investigated price games in which each firm sets its price and the maximum quantity it is willing to

Email address: atasnadi@axelero.hu (Attila Tasnádi).

URL: www.bkae.hu/ tasnadi (Attila Tasnádi).

1 I am grateful to Jan Boone, Eric van Damme, Ruud Hendrickx, Peter Kort, Miklós Pintér, András Simonovits, Dolf Talman, an anonymous referee, and seminar participants at CentER of Tilburg University and the University of Bonn for helpful comments and suggestions. This research was done during the author's Bolyai János Research Fellowship provided by the Hungarian Academy of Sciences (MTA). 
supply. In addition he discussed, but did not solve, the price-quantity game with both firms simultaneously choosing prices and quantities. Shubik (p. 430) conjectured that the value of the price-quantity game lies below that of the price game because of the risk of being left with inventory in the price-quantity game.

From now on, following Maskin's (1986) terminology, we will refer to the Shubik-type price game as a production-to-order game and to the Shubik-type price-quantity game as a production-in-advance game. Production in advance requires that production takes place before sales are realized. Markets of perishable goods are usually mentioned as examples of advance production in a market. In contrast, in case of production to order, sales are determined before production takes place. This mechanism is, for instance, typical in the market for ships or planes. Phillips et al. (2001) emphasized that there are also goods that can be traded both in a production-in-advance and in a production-toorder environment. To see this, we have to think about production-in-advance markets as a kind of spot market while production-to-order markets are a kind of forward market. Thus, for example, coal and electricity are sold in both types of environments. A comparison of these two different types of environments has been carried out in an experimental context by Mestelman et al. (1987) and Phillips et al. (2001). Assuming strictly increasing marginal cost functions, Mestelman et al. reports that in posted offer markets the firms' profits are lower in case of advance production, which is consistent with Shubik's conjecture. In an experimental auction market Phillips et al. found that prices and profits are higher while quantities and consumer surplus are lower in case of advance production.

We will compare production in advance with production to order in a theoretical framework. In order to keep our analysis tractable we will carry out the comparison in the framework of a homogeneous goods Bertrand-Edgeworth duopoly with capacity constraints. In the Bertrand-Edgeworth game quantities and prices are both decision variables. It is well known that in general this game does not have an equilibrium in pure strategies. The mixed-strategy equilibrium was computed, under different conditions imposed on the demand function and the capacity limits, by Beckmann (1965), Davidson and Deneckere (1986) as well as Allen and Hellwig (1993) for proportional rationing, and by Levitan and Shubik (1972), Kreps and Scheinkman (1983), Osborne and Pitchik (1986) and Vives (1986) for efficient rationing. Maskin demonstrated the existence of mixed-strategy equilibrium under very weak assumptions.

The above-mentioned works have determined mixed-strategy equilibrium corresponding to the case of production to order; that is, the production decisions of the firms follow the choices of their prices. Assuming unlimited capacities, Levitan and Shubik (1978) computed the mixed-strategy equilibrium for the 
case of production in advance (i.e., firms choose prices and production quantities simultaneously). However, if the firms are capacity constrained, then only the existence of a mixed-strategy equilibrium is known (see Maskin, Theorem 1 ). We are not aware of further theoretical results in the literature concerning the mixed-strategy equilibrium for the case of production in advance.

In this paper we consider the symmetric case in which both firms have equal capacities and the same constant unit costs up to their capacity constraints. We suppose that the unit costs are positive. This implies that in the two different cases, production to order and production in advance, the equilibrium in mixed strategies may differ. In particular, it seems possible that higher prices firms will not always produce up to their capacity constraints because they cannot sell their entire production with positive probability in a mixedstrategy equilibrium. We will establish that in a symmetric equilibrium the firms' profits are equal in both versions of the price-setting game (Theorem 1). This means that Shubik's conjecture does not hold true for the case of identical and constant unit costs up to the same capacity constraint. In addition, if both games' equilibrium are in nondegenerated symmetric mixed strategies, then the equilibrium price distribution of the production-in-advance case stochastically dominates (in the sense of first-order stochastic dominance) the equilibrium price distribution of the production-to-order case (Theorem 1).

The rest of this paper is organized as follows. Section 2 presents the framework of our analysis. Sections 3, 4 and 5 contain the comparison of the productionin-advance game with the production-to-order game for the case of small capacities, large capacities and intermediate capacities respectively. Finally, in Section 6 we make some concluding remarks.

\section{The model}

We impose the following assumptions on the demand curve $D: \mathbb{R}_{+} \rightarrow \mathbb{R}_{+}$.

Assumption $1 D$ is strictly decreasing on $[0, b]$, identically zero on $[b, \infty)$, continuous at $b$, twice continuously differentiable on $(0, b)$ and concave on $[0, b]$.

Note that a monopolist facing a demand curve satisfying Assumption 1, constant unit cost $c$ and positive capacity constraint $k$ has a unique positive profit maximizing price $p^{m}=\arg \max _{p \in[0, b]}(p-c) \min \{D(p), k\}$. We shall denote by $a$ the horizontal intercept of $D ; D(0)=a$.

In our model two firms set their prices and quantities. Regarding the duopolists we make the following assumptions: 
Assumption 2 The two firms, labelled 1 and 2, have identical positive unit costs $c \in(0, b)$ up to the same positive capacity constraint $k$. Each of them sets its price $\left(p_{1}, p_{2} \in[0, b]\right)$ and quantity $\left(q_{1}, q_{2} \in[0, k]\right)$.

The quantity decisions $q_{1}$ and $q_{2}$ shall be interpreted differently in case of production in advance and in case of production to order. In the first case $q_{i}$ stands for the actual production of firm $i$, while in the second case $q_{i}$ denotes the maximum amount firm $i$ is willing to supply. Throughout the paper $i$ and $j$ will be used to refer to the two firms; in particular, $i, j \in\{1,2\}$ and $i \neq j$.

In order to enable the demand faced by the firms to be determined, we have to specify a rationing rule. We assume efficient rationing by the low-price firm, which occurs in a market if the consumers can costlessly resell the good to each other or if the consumers have heterogeneous unit demands and the consumers having higher reservation prices are served first (for more details we refer to Davidson and Deneckere, 1986 and Tirole, 1988).

Assumption 3 The demand faced by firm $i$ is given by

$$
\Delta_{i}\left(p_{1}, q_{1}, p_{2}, q_{2}\right)= \begin{cases}D\left(p_{i}\right), & \text { if } p_{i}<p_{j} \\ \frac{q_{i}}{q_{i}+q_{j}} D\left(p_{i}\right), & \text { if } p_{i}=p_{j} \\ \left(D\left(p_{i}\right)-q_{j}\right)^{+}, & \text {if } p_{i}>p_{j} .\end{cases}
$$

In Assumption 3 three cases are considered. First, if firm $i$ is the low-price firm, then it faces the entire demand. Second, if they set the same price, then the demand is split in proportion of the firms' quantity decisions 1 Third, if firm $i$ sets the higher price, then it will face a so-called residual demand, which equals the demand minus the quantity produced by the low-price firm.

To specify the two games, we have to define the firms' profit functions. In case of production in advance, firm $i$ 's sales are constrained by its production $q_{i}$ or by its demand $\Delta_{i}\left(p_{1}, q_{1}, p_{2}, q_{2}\right)$. Moreover, setting quantity $q_{i}$ means that actual production equals $q_{i}$, which results in $c q_{i}$ cost. Thus, firm $i$ 's profit is given by

$$
\pi_{i}\left(\left(p_{1}, q_{1}\right),\left(p_{2}, q_{2}\right)\right)=p_{i} \min \left\{\Delta_{i}\left(p_{1}, q_{1}, p_{2}, q_{2}\right), q_{i}\right\}-c q_{i}
$$

in case of advance production. However, in case of production to order firm $i$ will never produce more than the demand it faces. Hence, its profit equals its

\footnotetext{
$\overline{1}$ It is worth noting that for the results obtained in this paper many other splitting rules can be allowed. The essential property of the rule employed here is that firm $i$ 's demand is strictly increasing in firm $i$ 's own quantity.
} 
gains per unit times its sales; that is,

$$
\pi_{i}\left(\left(p_{1}, q_{1}\right),\left(p_{2}, q_{2}\right)\right)=\left(p_{i}-c\right) \min \left\{\Delta_{i}\left(p_{1}, q_{1}, p_{2}, q_{2}\right), q_{i}\right\} .
$$

In this case it is optimal for firm $i$ to set quantity $q_{i}=k$ whenever $p_{i} \geq c$ and quantity $q_{i}=0$ whenever $p_{i}<c$ because of Assumption 2. Thus, the production-to-order game reduces to a simple price-setting game.

Observe that if a firm sets its price lower than $c$, then it will produce nothing. However, if a firm does not produce anything, it can set its price arbitrarily, and the price it sets is irrelevant. Furthermore, it can be easily checked that any price above $p^{m}$ is dominated. Therefore, we may assume in the following without loss of generality that the set of prices equals $\left[c, p^{m}\right]$.

Two special prices play a major role in the analysis. We define $p^{*}$ to be the price that clears the firms' aggregate capacity from the market if such a price exists, and zero otherwise. That is,

$$
p^{*}= \begin{cases}D^{-1}(2 k), & \text { if } D(0)>2 k \\ 0, & \text { if } D(0) \leq 2 k .\end{cases}
$$

The function

$$
\pi^{r}(p)=(p-c)(D(p)-k)
$$

equals a firm's residual profit whenever its opponent sells $k$ and $D(p) \geq k$. Let $\bar{p}=\arg \max _{p \in\left[c, p^{m}\right]} \pi^{r}(p)$. Clearly, $p^{*}$ and $\bar{p}$ are well defined whenever Assumptions 1 and 2 are satisfied.

In the Introduction we mentioned that the production-to-order game has already been solved in the literature. Kreps and Scheinkman established the uniqueness of equilibrium profits. From Osborne and Pitchik we even know that the production-to-order game has a unique solution. We shall denote the equilibrium profit of the production-to-order game by $\bar{\pi}$. The following result is due to Levitan and Shubik (1972), Osborne and Pitchik (1986) and Vives (1986):

Proposition 1 Under Assumptions 1, 2 and [3, the production-to-order game has a unique symmetric equilibrium. In particular, the following three cases emerge:

(1) If $p^{*} \geq \bar{p}$, then we have an equilibrium in pure strategies with both firms setting prices equal to the market clearing price (i.e., $p_{i}=p^{*}$ ).

(2) If $\bar{p}>\max \left\{p^{*}, c\right\}$, then there is only an equilibrium in nondegenerated mixed strategies with distribution function

$$
F(p)=\frac{(p-c) k-\bar{\pi}}{(p-c)(2 k-D(p))}
$$


for any $p \in[\underline{p}, \bar{p}]$, where $\underline{p}=c+\bar{\pi} / k$.

(3) If $D(c) \leq k$, then we have an equilibrium in pure strategies with both firms setting prices equal to unit cost (i.e., $p_{i}=c$ ).

Case 1 in Proposition 1 occurs if the firms are capacity constrained, while case 3 occurs if each firm can serve at any price the entire demand. For this reason we will refer to the first case as the small-capacity case and to the third case as the large-capacity case. Case 2 can be called the case of intermediate capacities in which there is only an equilibrium in nondegenerated mixed strategies. We will divide our analysis of the production-in-advance game into these three cases, where we will treat each case in a separate section.

\section{The case of small capacities}

First, we start with the case of small capacities, which is the only case with an equilibrium in pure strategies.

Proposition 2 Let Assumptions 1, 2 and 3 be fulfilled. Then if the production-in-advance version of the Bertrand-Edgeworth duopoly game has a Nash equilibrium in pure strategies, it is given by $p_{i}=p^{*}$ and $q_{i}=k(i \in\{1,2\})$. Furthermore, a Nash equilibrium in pure strategies exists if and only if $p^{*} \geq \bar{p}$.

Proof. No equilibrium can exist with $c \leq p_{i}<p_{j}$ because in this case, if $D\left(p_{i}\right)>k$ or $p_{i}=c$, firm $i$ will increase its price; and if $D\left(p_{i}\right) \leq k$ and $p_{i}>c$, firm $i$ produces $q_{i}=D\left(p_{i}\right)$ and therefore firm $j$ will reduce its price below $p_{i}$. Thus, in a pure-strategy equilibrium both firms must set the same price $p$. Observe that none of the firms will set a price lower than $p^{*}$. There cannot be an equilibrium with $p_{i}=p_{j}>\max \left\{p^{*}, c\right\}$ because in this case at least one firm can benefit from unilaterally undercutting its opponent price. If $p^{*}<c$, then $p_{i}=p_{j}=c$ cannot be an equilibrium since in this case we must have $q_{i}+q_{j} \leq D(c)$ in an equilibrium; therefore at least one firm can earn positive profit by unilaterally setting a price above $c$.

Finally, if $p^{*} \geq c$, we have to determine those conditions under which $p_{i}=p^{*}$ and $q_{i}=k$ is an equilibrium profile. Note that $p^{*} \geq c$ implies $\bar{p}>c$. Now if $p^{*} \geq c$, then condition

$$
\frac{\mathrm{d} \pi^{r}}{\mathrm{~d} p}\left(p^{*}\right)=\left(p^{*}-c\right) D^{\prime}\left(p^{*}\right)+D\left(p^{*}\right)-k \leq 0 \quad \Leftrightarrow \quad p^{*} \geq \bar{p}
$$

is necessary and sufficient for $p_{i}=p^{*}$ and $q_{i}=k$ being an equilibrium profile because of Assumptions 1 and 2 . 
By comparing Proposition 1 with Proposition 2, we can observe that the respective equilibrium prices and profits of the production-in-advance game and of the production-to-order game coincide whenever a pure-strategy equilibrium does exist in the production-in-advance game. In addition, Proposition 2 points out a difference between the production-in-advance and the productionto-order versions of the Bertrand-Edgeworth game since in the latter version an equilibrium in pure strategies also exists when capacities are sufficiently large. Assuming proportional rationing, Boyer and Moreaux (1987, Proposition 1) obtained a result in the same direction as Proposition 2 in that they showed the nonexistence of equilibrium in pure strategies for a price-setting production-in-advance game in case of unlimited capacities.

\section{The case of large capacities}

In the previous section we have considered the case in which an equilibrium in pure strategies exists. Now we turn to the cases corresponding to cases 2 and 3 appearing in Proposition 1 in which by Proposition 2 the productionin-advance game has only an equilibrium in nondegenerated mixed strategies. In particular, in this section we solve the case of large capacities while in the next section we will solve the case of intermediate capacities.

From Maskin (Theorem 1) we know that the production in advance game possesses an equilibrium in mixed strategies. In the following, a mixed strategy is a probability measure defined on the $\sigma$-algebra of Borel measurable sets on $S=\left[c, p^{m}\right] \times[0, k]$. A mixed-strategy equilibrium $\left(\mu_{1}^{*}, \mu_{2}^{*}\right)$ is determined by the following two conditions:

$$
\pi_{1}\left(\left(p_{1}, q_{1}\right), \mu_{2}^{*}\right) \leq \pi_{1}^{*}, \quad \pi_{2}\left(\mu_{1}^{*},\left(p_{2}, q_{2}\right)\right) \leq \pi_{2}^{*}
$$

holds true for all $\left(p_{1}, q_{1}\right),\left(p_{2}, q_{2}\right) \in S$, and

$$
\pi_{1}\left(\left(p_{1}^{*}, q_{1}^{*}\right), \mu_{2}^{*}\right)=\pi_{1}^{*}, \quad \pi_{2}\left(\mu_{1}^{*},\left(p_{2}^{*}, q_{2}^{*}\right)\right)=\pi_{2}^{*}
$$

holds true $\mu_{1}^{*}$-almost everywhere and $\mu_{2}^{*}$-almost everywhere, where $\pi_{1}^{*}, \pi_{2}^{*}$ stand for the equilibrium profits corresponding to $\left(\mu_{1}^{*}, \mu_{2}^{*}\right)$. The calculation of a mixed-strategy equilibrium in a closed form through conditions (2) and (3) is a very difficult and, perhaps, even impossible task. Therefore, we restrict ourselves to comparing the equilibrium of the production-to-order game with the equilibrium of the production-in-advance game. In addition, we will only consider symmetric mixed-strategy equilibrium, which simplifies the analysis and can be justified by the symmetric setting of the game. The existence of a symmetric equilibrium in mixed strategies can be established by applying Theorem $6^{*}$ in Dasgupta and Maskin (1986a). In fact, Maskin showed that 
$\pi_{1}+\pi_{2}$ is upper semi-continuous in case of advance production and Property $\left(\alpha^{*}\right)$ can be established similarly to the case of production to order (see Dasgupta and Maskin, 1986b).

Before we can proceed, we need some further notations. Let $\mu$ be a symmetric mixed-strategy equilibrium of the production-in-advance game. We shall denote the equilibrium profit of the production-in-advance game by $\tilde{\pi}_{\mu}$. We define the support of a symmetric equilibrium strategy $\mu$ in the following way:

$$
\operatorname{supp}(\mu)=\left\{(p, q) \in S \mid \pi_{1}((p, q), \mu)=\tilde{\pi}_{\mu}\right\} .
$$

For any price $p \in\left[c, p^{m}\right]$ we denote by $s_{\mu}(p) \subseteq[0, k]$ the set of those quantities $q \in[0, k]$ for which $(p, q) \in \operatorname{supp}(\mu)$. The correspondence $s_{\mu}$ may be called the supply correspondence. Let $\mu^{p}$ stand for the projection of probability measure $\mu$ to the set of prices; that is, $\mu^{p}(B)=\mu(B \times[0, k])$ for any Borel set $B \subseteq\left[c, p^{m}\right]$. We write $\widetilde{p}_{\mu}$ and $\widehat{p}_{\mu}$ for $\sup \left\{p \in\left[c, p^{m}\right] \mid \mu^{p}([c, p))=0\right\}$ and $\inf \left\{p \in\left[c, p^{m}\right] \mid \mu^{p}\left(\left(p, p^{m}\right]\right)=0\right\}$ respectively. Thus, $\mu^{p}\left(\left[\widetilde{p}_{\mu}, \widehat{p}_{\mu}\right]\right)=1$. In what follows we will omit the subscripts $\mu$ when it is clear which symmetric equilibrium strategy is meant, and we will write simply $\widetilde{\pi}, s, \widetilde{p}$ and $\widehat{p}$ instead of $\tilde{\pi}_{\mu}, s_{\mu}, \widetilde{p}_{\mu}$ and $\widehat{p}_{\mu}$ respectively.

From now on we will restrict ourselves in the analysis of the production-inadvance version of the game to those cases in which the equilibrium is in nondegenerated mixed strategies; that is, $p^{*}<\bar{p}$ by Proposition 2 . Clearly, $\tilde{\pi}_{\mu} \geq 0$ holds true since each firm can assure zero profit by zero production. Hence, $\widetilde{\pi}_{\mu}=0$ if $\widetilde{p}_{\mu}=c$. The following two lemmas investigate the case of $\tilde{p}_{\mu}>c$.

Lemma 1 If $(\mu, \mu)$ is a symmetric mixed-strategy equilibrium of the production-in-advance game, then under Assumptions 1, 2, 3, $p^{*}<\bar{p}$ and $\widetilde{p}_{\mu}>c$, we have $s_{\mu}\left(\widetilde{p}_{\mu}\right)=\left\{\min \left\{k, D\left(\widetilde{p}_{\mu}\right)\right\}\right\}$ and $\mu^{p}\left(\left\{\widetilde{p}_{\mu}\right\}\right)=0$.

Proof. Let $(\mu, \mu)$ be an arbitrary symmetric mixed-strategy equilibrium of the production-in-advance game. Suppose that $s(\widetilde{p})=\emptyset$. Clearly, there exists a sequence $p_{n}$ and $q_{n}$ such that $p_{n} \geq \widetilde{p},\left(p_{n}, q_{n}\right) \in \operatorname{supp}(\mu)$ and $\lim _{n \rightarrow \infty} p_{n}=\widetilde{p}$. For this sequence we have $\widetilde{\pi}=$

$$
\lim _{n \rightarrow \infty} \pi_{1}\left(\left(p_{n}, q_{n}\right), \mu\right) \leq \lim _{n \rightarrow \infty}\left(p_{n}-c\right) \min \left\{k, D\left(p_{n}\right)\right\}=(\widetilde{p}-c) \min \{k, D(\widetilde{p})\}
$$

Thus, $\tilde{\pi}=\pi_{1}((\widetilde{p}, \min \{k, D(\widetilde{p})\}), \mu)$, which is in contradiction with $s(\widetilde{p})=\emptyset$. Hence, $s(\widetilde{p}) \neq \emptyset$. If there exists a $q \in s(\widetilde{p})$ such that $q<\min \{k, D(\widetilde{p})\}$, then there exists an $\varepsilon>0$ for which

$$
\pi_{1}((\widetilde{p}, q), \mu) \leq(\widetilde{p}-c) q<(\widetilde{p}-\varepsilon-c) \min \{k, D(\widetilde{p}-\varepsilon)\}
$$


and thus, $(\mu, \mu)$ cannot be a mixed-strategy equilibrium. This means that $s(\widetilde{p})=\{\min \{k, D(\widetilde{p})\}\}$.

If $\mu^{p}$ has an atom at price $\widetilde{p}$, then it can be checked that the firms have to share the demand with positive probability whenever they play their pure strategy $(\widetilde{p}, \min \{k, D(\widetilde{p})\}) \in \operatorname{supp}(\mu)$, but then each firm can increase its profit by switching unilaterally to pure strategy $(\widetilde{p}-\varepsilon, \min \{k, D(\widetilde{p}-\varepsilon)\})$, where $\varepsilon$ is a sufficiently small positive value. However, this cannot be the case because $(\mu, \mu)$ is a mixed-strategy equilibrium, and thus, there is no atom at price $\widetilde{p}$.

From Lemma 1 we see that $\widetilde{\pi}_{\mu}=\left(\widetilde{p}_{\mu}-c\right) \min \left\{k, D\left(\widetilde{p}_{\mu}\right)\right\}$. Lemma 2 and Lemma 4 extend the results of Lemma 1 to the bottom part of the support of equilibrium prices. Lemma 2 considers the case in which the lowest possible price $\widetilde{p}_{\mu}$ is high enough so that the firms are demand constrained rather than capacity constrained; that is, $s_{\mu}\left(\widetilde{p}_{\mu}\right)=\left\{D\left(\widetilde{p}_{\mu}\right)\right\}$.

Lemma 2 If $(\mu, \mu)$ is a symmetric mixed-strategy equilibrium of the production-in-advance game, then under Assumptions 1, 2, 3, $p^{*}<\bar{p}, \widetilde{p}_{\mu}>c$ and $s_{\mu}\left(\widetilde{p}_{\mu}\right)=\left\{D\left(\widetilde{p}_{\mu}\right)\right\}$, there exists a price $p^{\prime} \in\left(\widetilde{p}_{\mu}, p^{m}\right]$ such that $s(p)=\{D(p)\}$ and $\mu^{p}(\{p\})=0$ for all $p \in\left[\widetilde{p}, p^{\prime}\right]$.

Proof. Let $(\mu, \mu)$ be an arbitrary symmetric mixed-strategy equilibrium of the production-in-advance game. First, observe that $D\left(p^{m}\right)<k$ must hold, since otherwise, $D\left(p^{m}\right)=k$, which in turn implies $p^{m} \leq \widetilde{p}<\widehat{p}$ by $s(\widetilde{p})=\{D(\widetilde{p})\}$, a contradiction. Note that we must have $\widetilde{p}<p^{m}$, because otherwise, $\widetilde{p}=p^{m}=\widehat{p}$ follows, which cannot be the case because of $\mu^{p}(\{\tilde{p}\})=0$. These observations will be useful in the remaining part of the proof.

Firm 1's profit, given that firm 2 plays its own equilibrium strategy $\mu$, equals

$$
\begin{aligned}
\pi_{1}((p, q), \mu)= & p \int_{[\widetilde{p}, p) \times[0, k]} \min \left\{\left(D(p)-q_{2}\right)^{+}, q\right\} d \mu\left(p_{2}, q_{2}\right)+ \\
& p \int_{\{p\} \times[0, k]} \min \left\{\frac{q}{q+q_{2}} D(p), q\right\} d \mu\left(p_{2}, q_{2}\right)+ \\
& p q \mu^{p}((p, \hat{p}])-c q,
\end{aligned}
$$

where $(p, q) \in \operatorname{supp}(\mu)$. Note that $(p, q) \in \operatorname{supp}(\mu)$ implies $q \leq D(p)$. Clearly, the two integrals in the above expression are both increasing in $q$. Therefore, $p \mu^{p}((p, \hat{p}])-c>0$ implies that $\pi_{1}$ is strictly increasing in $q$ whenever $p$ and $\mu$ are fixed. Hence, by $\widetilde{p}<p^{m}$ and Lemma 1 there exists a $p^{\prime} \in\left(\widetilde{p}, p^{m}\right]$ such that $s\left(p^{\prime}\right)=\left\{D\left(p^{\prime}\right)\right\}$, and $s(p)=\{D(p)\}$ or $s(p)=\emptyset$ for all $p \in\left[\widetilde{p}, p^{\prime}\right)$. 
By Lemma $1 \mu^{p}$ does not have an atom at $\widetilde{p}$. Suppose that $\mu^{p}$ has an atom at price $p \in\left(\widetilde{p}, p^{\prime}\right]$. Since $\mu^{p}$ has at most countably many atoms, we can select a sufficiently small $\varepsilon>0$ such that $p-\varepsilon>\widetilde{p}, \mu^{p}(\{p-\varepsilon\})=0$ and

$$
\begin{aligned}
\pi_{1}((p-\varepsilon, D(p-\varepsilon)), \mu) & =(p-\varepsilon) D(p-\varepsilon) \mu^{p}((p-\varepsilon, \widehat{p}])-c D(p-\varepsilon) \\
& \geq(p-\varepsilon) D(p-\varepsilon) \mu^{p}([p, \widehat{p}])-c D(p-\varepsilon) \\
& >p \frac{1}{2} D(p) \mu^{p}(\{p\})+p D(p) \mu^{p}((p, \widehat{p}])-c D(p) \\
& =\pi_{1}((p, D(p)), \mu) .
\end{aligned}
$$

Hence, $(p, D(p)) \notin \operatorname{supp}(\mu)$. However this is in contradiction with our assumption that $\mu^{p}$ has an atom at price $p$.

We establish that $s(p)=\{D(p)\}$ for all $p \in\left[\widetilde{p}, p^{\prime}\right]$. Suppose that the interval $[\alpha, \beta] \subset\left(\widetilde{p}, p^{\prime}\right)$, where $\alpha \leq \beta$, is a 'price gap' so that, for all $p \in[\alpha, \beta]$ we have $s(p)=\emptyset$ and we can take sequences $p_{n}$ and $p_{n}^{\star}$ of prices such that $p_{n} \in[\widetilde{p}, \alpha), s\left(p_{n}\right)=\left\{D\left(p_{n}\right)\right\}, \lim _{n \rightarrow \infty} p_{n}=\alpha, p_{n}^{\star} \in\left(\beta, p^{\prime}\right], s\left(p_{n}^{\star}\right)=\left\{D\left(p_{n}^{\star}\right)\right\}$ and $\lim _{n \rightarrow \infty} p_{n}^{\star}=\beta$. Then we must have

$$
\begin{aligned}
& \pi_{1}((\alpha, D(\alpha)), \mu)=\lim _{n \rightarrow \infty} \pi_{1}\left(\left(p_{n}, D\left(p_{n}\right)\right), \mu\right)=\tilde{\pi} \quad \text { and } \\
& \pi_{1}((\beta, D(\beta)), \mu)=\lim _{n \rightarrow \infty} \pi_{1}\left(\left(p_{n}^{\star}, D\left(p_{n}^{\star}\right)\right), \mu\right)=\tilde{\pi}
\end{aligned}
$$

since $\mu^{p}$ is atomless on $\left[\widetilde{p}, p^{\prime}\right]$. However this implies that $s(\alpha)=\{D(\alpha)\}$ and $s(\beta)=\{D(\beta)\}$, a contradiction. In an analogous way one can establish that 'price gaps' of type $[\alpha, \beta) \subset\left(\widetilde{p}, p^{\prime}\right)$ and $(\alpha, \beta] \subset\left(\widetilde{p}, p^{\prime}\right)$ cannot occur. Finally, suppose that the interval $(\alpha, \beta) \subseteq\left(\widetilde{p}, p^{\prime}\right)$ is a 'price gap'; that is, $s(\alpha)=\{D(\alpha)\}, s(\beta)=\{D(\beta)\}$ and $s(p)=\emptyset$ for all $p \in(\alpha, \beta)$. Then $\pi_{1}((\gamma, D(\gamma)), \mu)=\gamma D(\gamma) \mu^{p}([\beta, \widehat{p}])-c D(\gamma)$ is strictly increasing in $\gamma$ on $(\alpha, \beta)$ because of $\beta \leq p^{m}$ and Assumption 1, but this cannot happen because $\lim _{\gamma \rightarrow \alpha+0} \pi_{1}((\gamma, D(\gamma)), \mu)=\pi_{1}((\alpha, D(\alpha)), \mu)=\tilde{\pi}$ since $\mu^{p}$ is atomless on $\left[\widetilde{p}, p^{\prime}\right]$.

Suppose that $(\mu, \mu)$ is a symmetric mixed-strategy equilibrium. In the following let $p^{k}=$

$$
\sup \left\{p^{\prime} \in\left[\widetilde{p}, p^{m}\right] \mid \forall p \in\left[\widetilde{p}, p^{\prime}\right): s(p)=\{\min \{k, D(p)\}\} \text { and } \mu^{p}(\{p\})=0\right\} \text {. }
$$

Lemma 2 assures that $p^{k}>\widetilde{p}$ if Assumptions 1, 2, 3, $p^{*}<\bar{p}, \widetilde{p}>c$ and $s(\widetilde{p})=\{D(\widetilde{p})\}$ are fulfilled.

Lemma 3 If $(\mu, \mu)$ is a symmetric mixed-strategy equilibrium of the production-in-advance game, then under Assumptions 1, 2, 3, $p^{*}<\bar{p}$ and $s_{\mu}\left(\widetilde{p}_{\mu}\right)=$ $\left\{D\left(\widetilde{p}_{\mu}\right)\right\}$ we have $\widetilde{\pi}_{\mu}=0$. 
Proof. Take an arbitrary symmetric mixed-strategy equilibrium $(\mu, \mu)$ of the production-in-advance game. Clearly, Lemma 3 holds true if $\widetilde{p}=c$; therefore, in what follows we will assume that $\widetilde{p}>c$. If $s\left(p^{k}\right)=\emptyset$, then $\mu^{p}$ is atomless on $\left[\widetilde{p}, p^{k}\right]$ and thus, $\pi_{1}\left(\left(p^{k}, D\left(p^{k}\right)\right), \mu\right)=\lim _{p \rightarrow p^{k}-0} \pi_{1}((p, D(p)), \mu)=\widetilde{\pi}$, a contradiction. Hence, $s\left(p^{k}\right) \neq \emptyset$. By the definition of $p^{k}$ and Lemma 2, we must have $p \mu^{p}((p, \widehat{p}])-c \geq 0$ for all $p \in\left[\widetilde{p}, p^{k}\right)$. Thus,

$$
p^{k} \mu^{p}\left(\left[p^{k}, \widehat{p}\right]\right)-c=\lim _{p \rightarrow p^{k}-0} p \mu^{p}((p, \widehat{p}])-c \geq 0 .
$$

Let $H(q)=p^{k} q \mu^{p}\left(\left[p^{k}, \hat{p}\right]\right)-c q$. Obviously, $\pi_{1}\left(\left(p^{k}, q\right), \mu\right) \leq H(q)$ for all $q \in[0, k]$. We have $\tilde{\pi}=\pi_{1}((p, D(p)), \mu)$ for all $p \in\left[\widetilde{p}, p^{k}\right)$ by the definition of $p^{k}$ and Lemma 2, By applying the first part of (5), it can be verified that

$$
\widetilde{\pi}=\lim _{p \rightarrow p^{k}-0} \pi_{1}((p, D(p)), \mu)=H\left(D\left(p^{k}\right)\right) .
$$

Regarding (5) we consider two cases. First, we assume that $p^{k} \mu^{p}\left(\left[p^{k}, \widehat{p}\right]\right)-c=$ 0 . Then $0=H(q)=H\left(D\left(p^{k}\right)\right)=\tilde{\pi}$ for all $q \in\left[0, D\left(p^{k}\right)\right]$ by $(6)$. Thus, we have obtained a contradiction with $\widetilde{p}>c$, and therefore it follows that $\widetilde{p}=c$, which in turn implies $\widetilde{\pi}=0$.

Second, we assume that $p^{k} \mu^{p}\left(\left[p^{k}, \hat{p}\right]\right)-c>0$. Then $\mu^{p}\left(\left[p^{k}, \hat{p}\right]\right)>0$ and $\tilde{\pi}=$ $H\left(D\left(p^{k}\right)\right)>H(q) \geq \pi_{1}\left(\left(p^{k}, q\right), \mu\right)$ for all $q \in\left[0, D\left(p^{k}\right)\right)$. Thus, $s\left(p^{k}\right)=$ $\left\{D\left(p^{k}\right)\right\}$. It can be verified that the definition of $p^{k}$ and $p^{k} \mu^{p}\left(\left[p^{k}, \widehat{p}\right]\right)-c>0$ implies $\mu^{p}\left(\left\{p^{k}\right\}\right)>0$. Hence,

$$
\pi_{1}\left(\left(p^{k}, D\left(p^{k}\right)\right), \mu\right)<\pi_{1}\left(\left(p^{k}-\varepsilon, D\left(p^{k}-\varepsilon\right)\right), \mu\right)=\widetilde{\pi}
$$

for a sufficiently small $\varepsilon>0$, which is in contradiction with $s\left(p^{k}\right)=\left\{D\left(p^{k}\right)\right\}$. Therefore, the second case cannot occur.

Lemmas 1, 2 and 3 are valid for any case in which a pure-strategy equilibrium does not exist, that is, for large capacities as well as intermediate capacities. In fact, Lemmas 1 and 3 will be also applied in Section 5 where we will investigate the case of intermediate capacities. Now we separate our analysis into two parts: the case of $D(c) \leq k$ (large capacities) and the case of $\bar{p}>\max \left\{p^{*}, c\right\}$ (intermediate capacities). Proposition 2 already covers the case of $p^{*} \geq \bar{p}$. The following proposition contains our result for the case of $D(c) \leq k$.

Proposition 3 If $(\mu, \mu)$ is a symmetric mixed-strategy equilibrium of the production-in-advance game, then under Assumptions 1, 2, 3 and $D(c) \leq k$, we 
have $\tilde{\pi}_{\mu}=\bar{\pi}=0$.

Proof. From Proposition 11 we already know that the production-to-order game has an equilibrium in pure strategies with $\bar{\pi}=0$ and both firms setting their prices equal to their marginal costs $c$.

Now, we turn to the production-in-advance game. Of course, if $\widetilde{p}=c$, then $\tilde{\pi}=0$, and we are finished. If $\tilde{p}>c$, then we can apply Lemma 1. Note that $D(c) \leq k$ implies $s(\widetilde{p})=\{D(\widetilde{p})\}$. Therefore, we can apply Lemma 3 and obtain $\widetilde{\pi}=0$.

We can also observe that under the assumptions of Proposition 3 the production-in-advance prices dominate, in the sense of first-order stochastic dominance, the production-to-order prices, since in the production-in-advance game the firms never set prices below $c$ and in the production-to-order game the firms set prices equal to $c$. In addition, Proposition 3 is consistent with Levitan and Shubik (1978), since they also obtained that the firms make zero profits in case of advance production. However, they assumed the presence of unlimited capacities.

\section{The case of intermediate capacities}

In this Section we will consider the remaining case, that is, the case of $\bar{p}>$ $\max \left\{p^{*}, c\right\}$. We start with the observation that in this case each firm can guarantee itself $\bar{\pi}>0$ profits in the production-in-advance version of the game by setting its price to $\bar{p}$ and its quantity to $D(\bar{p})-k$. Thus, $\bar{\pi} \leq \tilde{\pi}$. This observation also implies that $\tilde{p} \geq \underline{p}>\max \left\{p^{*}, c\right\} \geq c$.

Before stating our result concerning the case of $\bar{p}>\max \left\{p^{*}, c\right\}$, we need another lemma, which is analogous to Lemma 2 in that it extends the result of Lemma 1 to the bottom part of the symmetric equilibrium price distribution.

Lemma 4 If $(\mu, \mu)$ is a symmetric mixed-strategy equilibrium of the production-in-advance game, then under Assumptions 1, 2, 3 and $\bar{p}>\max \left\{p^{*}, c\right\}$ we have $k<D\left(\widetilde{p}_{\mu}\right)$ and there exists a price $p^{\prime} \in\left(\widetilde{p}_{\mu}, p^{m}\right]$ such that $s(p)=\{k\}$ and $\mu^{p}(\{p\})=0$ for all $p \in\left[\widetilde{p}_{\mu}, p^{\prime}\right]$.

Proof. Let $(\mu, \mu)$ be an arbitrary symmetric mixed-strategy equilibrium of the production-in-advance game. First, observe that we must have $k<D(\widetilde{p})$ and $s(\widetilde{p})=\{k\}$ because $s(\widetilde{p})=\{D(\widetilde{p})\}$ would imply by Lemma 3 that $\widetilde{\pi}=0$, which cannot be the case since $0<\pi \leq \tilde{\pi}$. 
The two integrals in (4) are both increasing in $q$. Hence, $p \mu^{p}((p, \hat{p}])-c>0$ implies that $\pi_{1}$ is strictly increasing in $q$ whenever $p$ and $\mu$ are fixed. Then it can be checked that by Lemma 1 there exists a price $p^{\prime} \in\left(\widetilde{p}, p^{m}\right]$ such that $s\left(p^{\prime}\right)=\{k\}$, and $s(p)=\{k\}$ or $s(p)=\emptyset$ for all $p \in\left[\widetilde{p}, p^{\prime}\right]$.

We already know by Lemma 1 that $\mu^{p}$ does not have an atom at $\widetilde{p}$. Suppose that $\mu^{p}$ has an atom at price $p \in\left(\widetilde{p}, p^{\prime}\right]$. Then it follows that

$$
k \mu^{p}(\{p\})>\frac{1}{2} D(p) \mu^{p}(\{p\}) .
$$

Since $\mu^{p}$ has at most countably many atoms we can select a sufficiently small $\varepsilon>0$ such that $p-\varepsilon>\widetilde{p}, \mu^{p}(\{p-\varepsilon\})=0$ and

$$
\begin{aligned}
\pi_{1}((p-\varepsilon, k), \mu)= & (p-\varepsilon) \min \left\{(D(p-\varepsilon)-k)^{+}, k\right\} \mu^{p}([\widetilde{p}, p-\varepsilon))+ \\
& (p-\varepsilon) k \mu^{p}((p-\varepsilon, \widehat{p}])-c k \\
\geq & (p-\varepsilon) \min \left\{(D(p)-k)^{+}, k\right\} \mu^{p}([\widetilde{p}, p-\varepsilon))+ \\
& (p-\varepsilon) k \mu^{p}([p-\varepsilon, p))+(p-\varepsilon) k \mu^{p}(\{p\})+ \\
& (p-\varepsilon) k \mu^{p}((p, \widehat{p}])-c k \\
\geq & (p-\varepsilon) \min \left\{(D(p)-k)^{+}, k\right\} \mu^{p}([\widetilde{p}, p))+ \\
& (p-\varepsilon) k \mu^{p}(\{p\})+(p-\varepsilon) k \mu^{p}((p, \widehat{p}])-c k \\
> & p \min \left\{(D(p)-k)^{+}, k\right\} \mu^{p}([\widetilde{p}, p))+ \\
& p \frac{1}{2} D(p) \mu^{p}(\{p\})+p k \mu^{p}((p, \widehat{p}])-c k \\
= & \pi_{1}((p, k), \mu),
\end{aligned}
$$

where in the last inequality we applied (7). Hence, $(p, k) \notin \operatorname{supp}(\mu)$, a contradiction.

Finally, we show that $s(p)=\{k\}$ for all $p \in\left[\widetilde{p}, p^{\prime}\right]$. This holds true for $\widetilde{p}$ by Lemma 1. Suppose that the interval $[\alpha, \beta] \subset\left(\widetilde{p}, p^{\prime}\right)$, where $\alpha \leq \beta$, is a 'price gap'; that is, for all $p \in[\alpha, \beta]$ we have $s(p)=\emptyset$ and we can take sequences $p_{n}$ and $p_{n}^{\star}$ of prices such that $p_{n} \in[\widetilde{p}, \alpha), s\left(p_{n}\right)=\{k\}, \lim _{n \rightarrow \infty} p_{n}=\alpha$, $p_{n}^{\star} \in\left(\beta, p^{\prime}\right), s\left(p_{n}^{\star}\right)=\{k\}$ and $\lim _{n \rightarrow \infty} p_{n}^{\star}=\beta$. Then we must have

$$
\begin{aligned}
& \pi_{1}((\alpha, k), \mu)=\lim _{n \rightarrow \infty} \pi_{1}\left(\left(p_{n}, k\right), \mu\right)=\widetilde{\pi} \quad \text { and } \\
& \pi_{1}((\beta, k), \mu)=\lim _{n \rightarrow \infty} \pi_{1}\left(\left(p_{n}^{\star}, k\right), \mu\right)=\widetilde{\pi}
\end{aligned}
$$

since $\mu^{p}$ is atomless on $\left[\widetilde{p}, p^{\prime}\right]$, but this implies that $s(\alpha)=\{k\}$ and $s(\beta)=\{k\}$, a contradiction. In an analogous way one can establish that gaps of type $[\alpha, \beta) \subset\left(\widetilde{p}, p^{\prime}\right)$ and $(\alpha, \beta] \subset\left(\widetilde{p}, p^{\prime}\right)$ cannot occur. Now suppose that the interval $(\alpha, \beta) \subseteq\left(\widetilde{p}, p^{\prime}\right)$ is a 'price gap'; that is, $s(\alpha)=\{k\}, s(\beta)=\{k\}$ and $s(p)=$ 
$\emptyset$ for all $p \in(\alpha, \beta)$. Clearly, $\lim _{\gamma \rightarrow \alpha+0} \pi_{1}((\gamma, k), \mu)=\pi_{1}((\alpha, k), \mu)$ since $\mu^{p}(\{\alpha\})=0$, and $\lim _{\gamma \rightarrow \beta-0} \pi_{1}((\gamma, k), \mu)=\pi_{1}((\beta, k), \mu)$ since $\mu^{p}(\{\beta\})=0$. We must have $\mu^{p}([\widetilde{p}, \alpha])>0$ by the definition of $\widetilde{p}$. Hence, it can be verified that $\mu^{p}([\widetilde{p}, \alpha])>0$, implying that $\pi_{1}((\gamma, k), \mu)$ is strictly concave on $[\alpha, \beta]$ in $\gamma$ because of Assumption 1 and therefore, $\pi_{1}((\gamma, k), \mu)$ has a unique maximizer in $[\alpha, \beta]$. We conclude that $s(\alpha)=\emptyset$ or $s(\beta)=\emptyset$, a contradiction.

Lemma 4 guarantees that $p^{k}>\widetilde{p}$ if $\bar{p}>\max \left\{p^{*}, c\right\}$, Assumptions 1, 2 and 3 are satisfied.

Now, we state our result on symmetric mixed-strategy equilibrium for the case of $\bar{p}>\max \left\{p^{*}, c\right\}$ :

Proposition 4 Let Assumptions 1, 2 and 3 be satisfied. If $\bar{p}>\max \left\{p^{*}, c\right\}$, then for any symmetric mixed-strategy equilibrium $(\mu, \mu)$ of the production-inadvance game, we have $\widetilde{p}_{\mu}=\underline{p}, p^{k}=\bar{p}, \widetilde{\pi}_{\mu}=\bar{\pi}$ and

$$
\mu^{p}([\underline{p}, p))=\mu([\underline{p}, p),\{k\})=\frac{(p-c) k-\bar{\pi}}{p(2 k-D(p))}
$$

for any $p \in[\underline{p}, \bar{p}]$.

Proof. Take an arbitrary symmetric mixed-strategy equilibrium $(\mu, \mu)$ of the production-in-advance game. If $s\left(p^{k}\right)=\emptyset$, then $\mu^{p}$ is atomless on $\left[\widetilde{p}, p^{k}\right]$ and therefore,

$$
\pi_{1}\left(\left(p^{k}, \min \left\{k, D\left(p^{k}\right)\right\}\right), \mu\right)=\lim _{p \rightarrow p^{k}-0} \pi_{1}((p, \min \{k, D(p)\}), \mu)=\widetilde{\pi},
$$

a contradiction. Hence, $s\left(p^{k}\right) \neq \emptyset$. By the definition of $p^{k}$ and Lemma 4 we must have $p \mu^{p}((p, \widehat{p}])-c \geq 0$ for all $p \in\left[\widetilde{p}, p^{k}\right)$. Let

$$
G(q)=p^{k} q \mu^{p}\left(\left[p^{k}, \widehat{p}\right]\right)+p^{k}\left(D\left(p^{k}\right)-k\right)^{+} \mu^{p}\left(\left[\widetilde{p}, p^{k}\right)\right)-c q .
$$

Obviously, $\pi_{1}\left(\left(p^{k}, q\right), \mu\right) \leq G(q)$ for all $q \in\left[0, \min \left\{k, D\left(p^{k}\right)\right\}\right]$. We have $\widetilde{\pi}=\pi_{1}((p, \min \{k, D(p)\}), \mu)$ for all $p \in\left[\widetilde{p}, p^{k}\right)$ by Lemma 4 . In addition, by applying the first part of (5) it can be verified that

$$
\widetilde{\pi}=\lim _{p \rightarrow p^{k}-0} \pi_{1}((p, \min \{k, D(p)\}), \mu)=G\left(\min \left\{k, D\left(p^{k}\right)\right\}\right) .
$$

In order to prove that $p^{k}=\bar{p}$ we split (5) into two separate cases. First, we suppose that $p^{k} \mu^{p}\left(\left[p^{k}, \hat{p}\right]\right)-c=0$. Then $G(q)=G\left(\min \left\{k, D\left(p^{k}\right)\right\}\right)=\tilde{\pi}$ 
for all $q \in\left[0, \min \left\{k, D\left(p^{k}\right)\right\}\right]$ by $(9)$. Specially, if $k<D\left(p^{k}\right)$, then $\tilde{\pi}=$ $G\left(D\left(p^{k}\right)-k\right)=\pi_{1}\left(\left(p^{k}, D\left(p^{k}\right)-k\right), \mu\right) \leq \bar{\pi}$, which implies $\tilde{\pi}=\bar{\pi}, \tilde{p}=\underline{p}$ and $p^{k}=\bar{p}$. If $k \geq D\left(p^{k}\right)$, then $\tilde{\pi}=G(0)=0$. Thus, $k \geq D\left(p^{k}\right)$ cannot be the case since $\bar{p}>\max \left\{p^{*}, c\right\}$ implies $\widetilde{\pi}>0$.

Second, we suppose that $p^{k} \mu^{p}\left(\left[p^{k}, \widehat{p}\right]\right)-c>0$. Then

$$
\tilde{\pi}=G\left(\min \left\{k, D\left(p^{k}\right)\right\}\right)>G(q) \geq \pi_{1}\left(\left(p^{k}, q\right), \mu\right)
$$

for all $q \in\left[0, \min \left\{k, D\left(p^{k}\right)\right\}\right)$. Thus, $s\left(p^{k}\right)=\left\{\min \left\{k, D\left(p^{k}\right)\right\}\right\}$. Clearly, $\mu^{p}\left(\left[p^{k}, \widehat{p}\right]\right)>0$. It can be verified that the definition of $p^{k}$ and $p^{k} \mu^{p}\left(\left[p^{k}, \widehat{p}\right]\right)-$ $c>0$ implies $\mu^{p}\left(\left\{p^{k}\right\}\right)>0$. Hence, we obtain

$$
\pi_{1}\left(\left(p^{k}, \min \left\{k, D\left(p^{k}\right)\right\}\right), \mu\right)<G\left(\min \left\{k, D\left(p^{k}\right)\right\}\right)=\widetilde{\pi}
$$

by $\left(9\right.$, , which is in contradiction with $s\left(p^{k}\right)=\left\{\min \left\{k, D\left(p^{k}\right)\right\}\right\}$. Therefore, the second case cannot occur.

Now from Lemma 4 firm 1's profit, given that firm 2 plays its own equilibrium strategy $\mu$, equals

$$
\pi_{1}((p, k), \mu)=p k \mu^{p}((p, \widehat{p}])+p(D(p)-k) \mu^{p}([\widetilde{p}, p))-c k
$$

if $p \in\left[\widetilde{p}, p^{k}\right)$. Rearranging $10 p$ yields 8 since we have already established $\widetilde{p}=\underline{p}$ and $p^{k}=\bar{p}$.

Proposition 4 states that both the production-in-advance version and the production-to-order version of the capacity-constrained Bertrand-Edgeworth duopoly game have identical profits in a symmetric equilibrium. Furthermore, by comparing (1) with (8), we see that any symmetric equilibrium price distribution of the production-in-advance game stochastically dominates (in the sense of first-order stochastic dominance) the equilibrium price distribution of the production-to-order game. Thus, the payoff equivalence is even more surprising, since higher prices in case of production in advance result exactly in the same profits as in case of production to order. From Proposition 4 we conclude that under the assumptions of Proposition 4 the consumers' surplus in the production-in-advance game is smaller than in the production-to-order game, and therefore, the production-in-advance game leads to less welfare. This means that in the current setting from a social point of view production to order shall be preferred to advance production.

Propositions 2, 3 and 4 demonstrate the payoff equivalence of the productionin-advance game and the production-to-order game for any symmetric equilib- 
rium in case of symmetric capacities and identical unit costs. In addition, we found that the production-in-advance equilibrium prices stochastically dominate the production-to-order equilibrium prices. We state these results, which are our main results, in a theorem:

Theorem 1 Under Assumptions 1, 2 and 3, the production-in-advance profits and the production-to-order profits are the same, and the production-inadvance equilibrium prices dominate the production-to-order equilibrium prices if we restrict ourselves to symmetric equilibrium.

\section{Concluding remarks}

Our main result (Theorem 1) stating the equivalence of payoffs in case of production in advance and production to order differs from the experimental results by Mestelman et al. (1987) and Phillips et al. (2001). Mestelman et al. reported that advance production results in less profits than production to order, while Phillips et al. reported the opposite relationship. In addition, regarding the equilibrium price distributions, our Proposition 4 is consistent with Phillips et al. in that production in advance results in higher prices than production to order. In contrast Mestelman et al. observed lower prices in case of production in advance. Of course, this does not contradict our theoretical result because, among other reasons, the cited experimental works assumed increasing marginal costs, four sellers and four buyers in the market. It would be nice to carry out the comparison of payoffs for the case of increasing marginal costs in the theoretical framework, but this seems to be a very difficult task.

From Proposition 2 we know that the production-in-advance version of the capacity-constrained Bertrand-Edgeworth duopoly with identical capacity constraints and identical positive unit costs only possesses a Nash equilibrium in pure strategies if the firms' capacities are small. This disproves the statement that for small and large capacities the Bertrand-Edgeworth game has an equilibrium in pure strategies, while for an intermediate range of capacities there exists only an equilibrium in nondegenerated mixed strategies, which is usually associated with Bertrand-Edgeworth games.

\section{References}

Allen, B., Hellwig, M., 1993. Bertrand-Edgeworth duopoly with proportional demand. International Economic Review 34, 39-60.

Beckmann, M.J., with the assistance of Hochstädter, D., 1965. BertrandEdgeworth duopoly revisited. In: R. Henn (Ed.). Operations Research-Verfahren, Vol. III. Hain, Meisenheim, pp. 55-68. 
Boyer, M., Moreaux, M., 1987. Being a leader or a follower: reflections on the distribution of roles in duopoly. International Journal of Industrial Organization 5, 175-192.

Dasgupta, P., Maskin, E., 1986a. The existence of equilibria in discontinuous games I: theory. Review of Economic Studies 53, 1-26.

Dasgupta, P., Maskin, E., 1986b. The existence of equilibria in discontinuous games II: applications. Review of Economic Studies 53, 27-41.

Davidson, C., Deneckere, R., 1986. Long-run competition in capacity, shortrun competition in price, and the Cournot model. Rand Journal of Economics 17, 404-415.

Kreps, D.M., Scheinkman, J.A., 1983. Quantity precommitment and Bertrand competition yield Cournot outcomes. Bell Journal of Economics 14, 326-337.

Levitan, R., Shubik, M., 1972. Price duopoly and capacity constraints. International Economic Review 13, 111-122.

Levitan, R., Shubik, M., 1978. Duopoly with price and quantity as strategic variables. International Journal of Game Theory 7, 1-11.

Maskin, E., 1986. The existence of equilibrium with price-setting firms. American Economic Review 76, 382-386.

Mestelman, S., Welland, D., Welland, D., 1987. Advance production in posted offer markets. Journal of Economic Behavior and Organization 8, 249-264.

Osborne, M.J., Pitchik, C., 1986. Price competition in a capacity-constrained duopoly. Journal of Economic Theory 38, 238-260.

Phillips, O.R., Menkhaus, D.J, Krogmeier, J.L., 2001. Production-to-order or production-to-stock: the endogenous choice of institution in experimental auction markets. Journal of Economic Behavior and Organization 44, 333345 .

Shubik, M., 1955. A comparison of treatments of a duopoly problem (part II). Econometrica 23, 417-431.

Tirole, J., 1988. The Theory of Industrial Organization. MIT Press, Cambridge.

Vives, X., 1986. Rationing rules and Bertrand-Edgeworth equilibria in large markets. Economics Letters 21, 113-116. 